\title{
Looped-chain-based active current sharing strategy in DC microgrids
}

\author{
DEXIONG Li ${ }^{1}$, YiQI LIU ${ }^{2}$, Yong Gu${ }^{2}$, HUIJUAN QI ${ }^{2}$ \\ ${ }^{1}$ Shijiazhuang Institute of Railway Technology \\ ${ }^{2}$ Northeast Forestry University \\ Email liuyq0925@126.com
}

(Received: 15.12.2016, revised: 21.03.2017)

\begin{abstract}
The integration of renewable energy sources in modern electric grids have drawn increasing attention nowadays. In order to effectively manage large-scale renewable energy sources and achieve flexible and efficient operation, the concept of microgrids have been proposed. Considering the nature of DC outputs in many distributed energy resources (DERs), DC microgrids have been extensively studied in the past years. Among the operational issues in DC microgrids, current sharing issues have become an important topic since it is highly relevant to the operation of DC microgrids. By adopting a proper design of current sharing strategy in DC microgrids, the current rating violations in each interface converter can be successfully avoided. In this paper, a looped-chainbased active current sharing strategy is proposed to realize high accuracy current sharing in DC microgrids. In particular, the output current is shared between the neighboring interface converters. Hence, following a clockwise or counter-clockwise order, a loopedchain-based control diagram can be established to share the reference value of the output current. A final status in the whole DC microgrid is that the output current of every interface converter is equalized. Hence, the desired current sharing objective can be satisfied. A MATLAB simulation model is established to verify the proposed loopedchain-based active current sharing strategy in DC microgrids.
\end{abstract}

Key words: active current sharing, DC microgrids, looped control diagram

\section{Introduction}

The existing issues of conventional fossil fuels have urged the evolution of traditional power systems. The centralized generation pattern cannot fully satisfy the requirements of clean energy and $\mathrm{CO}_{2}$ emission. It is necessary to seek for the alternative energy sources that feature the characteristics of environment friendly, low or even none greenhouse gas emission, sustainable, etc. In order to meet these requirements, renewable energy sources have been of significant interest in the past years [1-3]. These types of energy sources are different from conventional fossil-fuel-based sources considering their advanced features. Typical renewable 
energy sources include photovoltaics (PVs), wind turbines, small hydro, etc. These renewable energy sources are common interconnected into distribution systems in a distributed manner. Hence, they are usually called distributed energy resources (DERs).

In order to solve the operational issues induced by increasing DER penetration level, people have proposed microgrids many years ago [4]. A microgrid is an effective solution to aggregate different types of distributed sources and loads and integrated them in a localized and small-scale power system.

Note that there are many types of DERs that feature DC outputs. These DERs include PVs, battery energy storages, etc. Meanwhile, many loads can be powered using DC common coupling, including LED lighting, DC motor, etc. Considering the above facts, it is a nature idea to integrate different types of DC-coupled DERs and loads using DC common bus. Therefore, a DC microgrid is thereby established [5-6]. Compared to AC microgrids, DC microgrid is coupled using DC bus, as shown in the typical configuration in Fig. 1. Hence, there are no issues regarding reactive power and harmonics since the whole system relies on DC electricity. At the same time, normally there are fewer converter interface needed in DC microgrids since energy conversion chain is shortened. This can be shown in the comparison between AC microgrids and DC microgrids, as shown in Fig. 2. Due to the shortened energy conversion chain, the system efficiency in DC microgrids can be enhanced compared to AC microgrids.

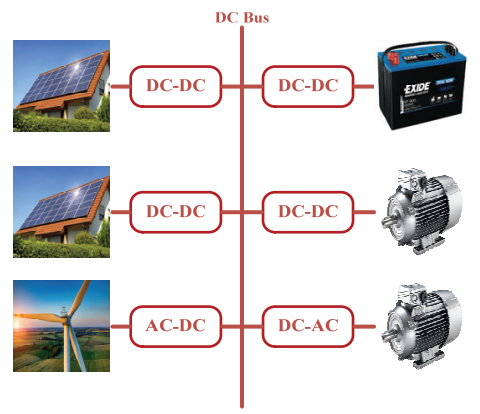

Fig. 1. Typical DC microgrid

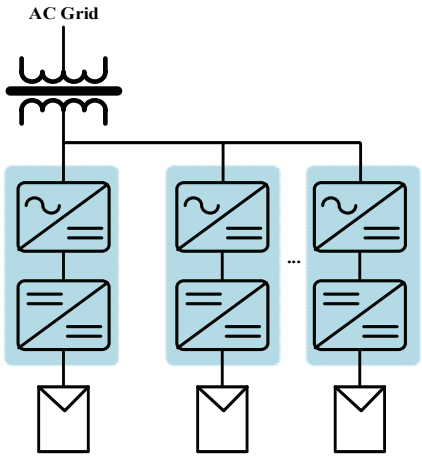

(a)

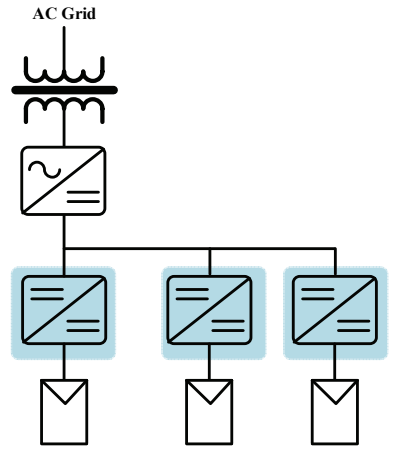

(b)

Fig. 2. Comparison of AC and DC microgrid configurations: AC microgrid (a); DC microgrid (b) 
Although there are many advantages in DC micorgrids, there are still some technical issues that need to be resolved [7]. The first issue in DC microgrids is active current sharing. The existence of line resistance can degrade the active current sharing in DC microgrids, which can be further deteriorated if the scale and physical size of a DC microgrid increase. Meanwhile, the second issue in DC microgrids is voltage deviation. Droop control is commonly used in DC microgrids to achieve load current sharing. Since the droop control method is used, it is necessary to realize that there is a voltage deviation imposed at the local or common DC bus voltage. This voltage deviation becomes larger with the increasing load current. It is necessary to eliminate the DC voltage deviation to further enhance the DC voltage stability.

In this paper, focusing on the above existing issues, a looped-chained-based current sharing algorithm is proposed to improve the active current sharing performance in DC microgrids. Particularly, by sharing the output current information among the neighboring interface converters in a DC micogrid, the reference value of each given interface converter is selected following the output current of its neighboring interface converter. Therefore, since this is applied to each converter, the output current in each converter can be finally equalized and the active current sharing performance can be enhanced. In the meanwhile, this proposed loopedchain-based active current sharing algorithm is in coordination with the common control diagram implemented in the central controller. These central control diagrams are used to restore the voltage level at the specific part of the DC microgrids and manage the power exchange between the local individual DC microgrids and external distribution systems.

The following papers are summarized as follows. Section II summarized the existing current sharing methods used in DC microgrids. Section III explains the technical details regarding the proposed looped-chain-based active current sharing strategy and its coordination with voltage restoration schemes and the power exchange control between local DC microgrids and external AC-coupled distribution systems. Meanwhile, the resilient operation of the proposed active current sharing algorithm by utilizing the redundant communication channels is explained in the proposed control algorithm. Section IV exhibits the simulation results based on the test model implemented in Simulink environment. Finally, Section V wraps up the paper and draws the conclusion.

\section{Review of active current sharing strategies in DC microgrids}

As aforementioned, active current sharing is an important issue in the operation of DC microgrids. This issue is particularly induced by the different values of line resistances connected to each DER [8]. As shown in Fig. 3, the line resistances in each branch are denoted as $R_{l 1}, R_{l 2}, \ldots, R_{l n}$, respectively. It can be derived that:

$$
v_{i}-i_{o i} \cdot r_{l i}=v_{p c c},
$$

where: $v_{i}$ is the output voltage of the interface converter $\# i, v_{p c c}$ is the voltage at the point of common coupling (PCC), $i_{o i}$ is the output current of the interface converter \#i. Since the whole DC microgrid is within the same DC voltage level, it is assumed that: 


$$
v_{1} \approx v_{2} \approx \cdots \approx v_{i} \approx \cdots \approx v_{n} .
$$

Therefore, it yields that:

$$
i_{o i} r_{l i}=C, \quad i=1,2, \ldots, n,
$$

where: $i_{o i}$ represents the output current of the $i^{\text {th }}$ converter, $r_{l i}$ represents the line impedance connected with the $i^{\text {th }}$ converter, and $\mathrm{C}$ represents the constant product of $i_{\mathrm{i} o}$ and $r_{l i}$.

As it is shown in (3), different line resistances impose the error in active current sharing in DC microgrids.

Various types of current sharing algorithms have been proposed in the existing work. In [9], graph theory and consensus algorithm are employed to achieve coordinated control and operation of multiple team-oriented converters. Since only local information is needed, the proposed method does not rely on central controller and does not need the total number of converters, which make it suitable for the plug-and-play operation of multiple DC-DC converters. In [10], rather than regulating DC voltage and applying consensus-based approach on current sharing, the droop coefficient of each converter is adjusted to further enhance the current sharing among multiple converters. In [11], a generalized system-level model comprised of multiple parallel converters is derived to analyze the power sharing issues considering line impedance. Meanwhile, adaptive gain scheduling technology is employed to compensate the mismatching line impedance.

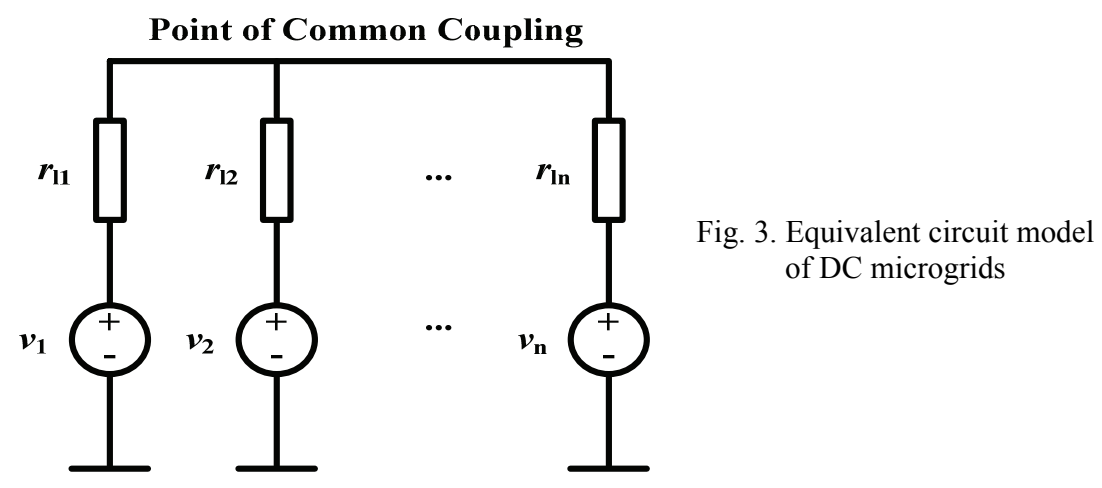

\section{Proposed looped-chain-based active current sharing strategy}

In order to achieve active current sharing in DC microgrids, a looped-chain-based approach is proposed in this paper. The overall control diagram is shown in Fig. 4. Rather than using a dedicated communication network for sharing DC output current measurements among all the interface converters in the DC microgrid, only localized communication network is established among neighboring converters. Additional controllers are employed for current sharing enhancement and voltage restoration. The established local complementary communication network is used for data exchange used in these two controllers. Meanwhile, 
the conventional droop controller and inner voltage and current controllers are also used in the proposed control diagram. In the additional control loop for active current sharing, the reference value of converter $\# i$ is set based on the DC output current of converter $\#(i+1)$. Hence, when selecting $i=1,2, \ldots, n$ in a DC microgrid with $\mathrm{n}$ interface converters, a looped architecture is established. In this proposed control architecture, since the proportional-integral (PI) controller is used in each additional control loop, the steady-state error of the DC output current between the neighboring interface converters can be gradually equalized. Therefore, it can be derived that:

$$
i_{o i+1}=i_{o i}
$$

where $i=1,2, \ldots, n-1$.

In (4), it is indicated that equal active current sharing can be enhanced in DC microgrids.

Compared to the conventional approaches, the proposed current sharing method features low communication dependency. As shown in the existing approaches, in order to improve the active current sharing in DC microgrids, communication networks, no matter dedicated high bandwidth communication network or complementary low bandwidth communication network, are usually needed. It is because that for each interface converter, at least the output current measurements in the neighboring units are needed so that the output current information in the whole DC microgrid can be obtained. To minimize the interdependency between the communication network and the proposed control systems, the looped configuration is employed in this study, which is only implemented using the DC output current measurement from the interface converter at one side. Hence, compared to the conventional approaches that at least rely on the DC output currents at both sides of the given interface converter, the communication amount can be halved.

In order to further improve the reliability of the proposed approaches, it should also be noted that the proposed secondary control scheme also has the degree of freedom to select from which side the DC output current is measured. In other words, the system control redundancy is improved by using one of the neighboring interface converter as the backup for obtaining DC output current measurement. As shown in Fig. 5, it can be clearly seen that the current measurements can be obtained from either neighboring converter. Fig. 5(a) and (b) shows the directions for transferring DC output current measurements, while in Fig. 5(c) and (d), additional paths that can be used for current measurement exchange are illustrated. As shown in Fig. 5(c) and (d), when some of the communication paths are damaged, the flexible communication network can bypass the faulty channel and alternative communication paths can be used to ensure continuous data exchange.

Rather than only focusing on active current sharing in DC microgrids, the voltage restoration can be also integrated in the proposed control diagram. Also as shown in Fig. 4, it can be seen that the secondary controller, which is commonly used in DC microgrids [12], is also employed the DC microgrid under study. However, for conventional secondary control diagram, it focuses on either the voltage restoration performance at the point of common coupling (PCC) in a DC microgrid or the output voltage of all the interface converters. This is generally a feasible solution in the operation of DC microgrids, while for some operation conditions, it 
is sometimes necessary to ensure the required control performance at the critical load buses. Hence, the selectivity of secondary control should be enhanced. Based on this fact, a selective secondary control scheme is proposed in this work, where the DC voltage at the selected buses are measured and averaged in the secondary controller. In the meanwhile, this averaged DC output voltage at all the selected buses is controlled to be restored to the rated value of the system. The set of the selected buses can be altered in real-time and the implementation of this secondary control scheme is managed in the control center of a DC microgrid. By using this proposed secondary control scheme, the flexible operation framework of restoring DC voltage at the buses with critical loads can be significantly enhanced.

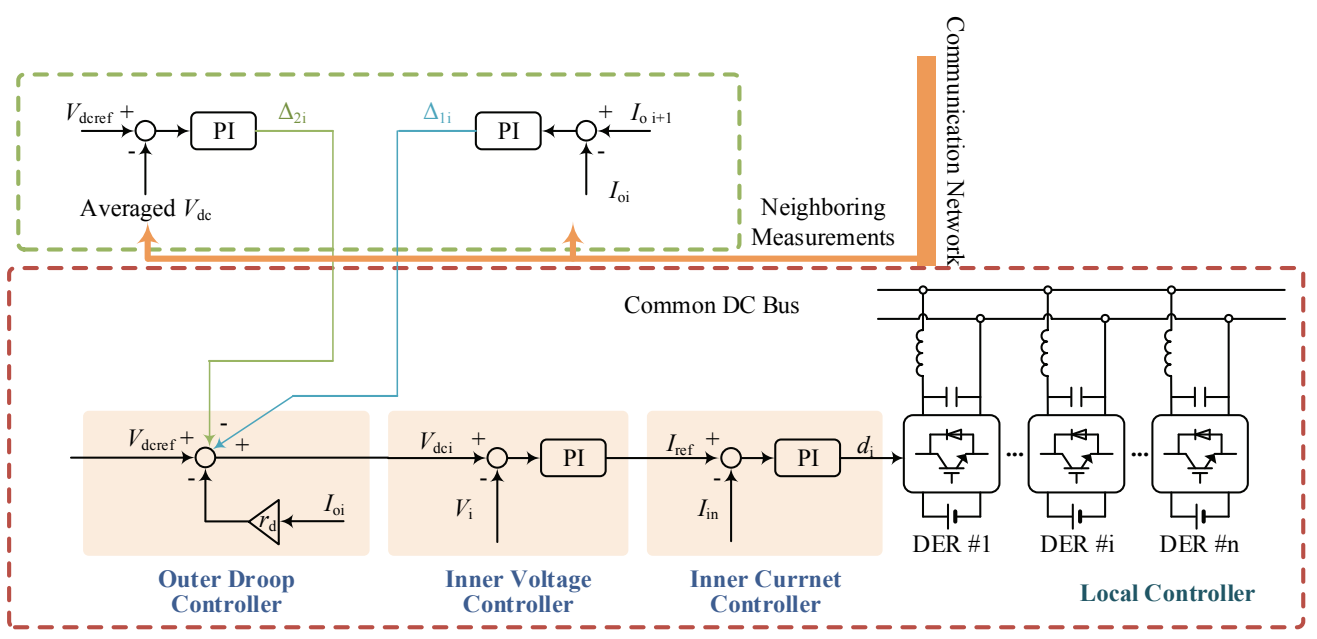

Fig. 4. Control diagram of the proposed approach

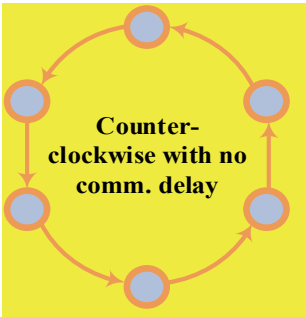

(a)

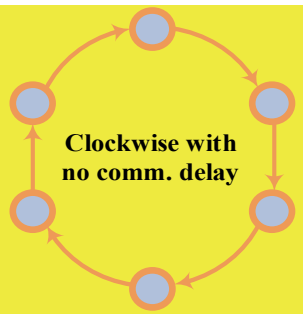

(b)

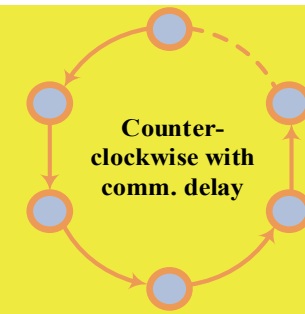

(c)

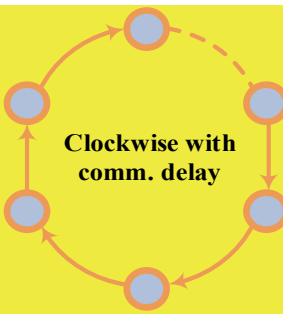

(d)

DER

Complete Communication Path

- - - Damaged Communication Path

Fig. 5. Communication paths using the proposed current sharing approach

Counter-clockwise data exchange without communication delay (a), clockwise data exchange without communication delay (b), counter-clockwise data exchange with communication delay (c), clockwise data exchange with communication delay (d). 


\section{Tests and verifications of the proposed current sharing strategy}

In order to verify the proposed looped-chain-based active current sharing approach and DC voltage restoration scheme for selective critical load buses, a test system with eight DC buses is implemented in Simulink environment. As shown in Fig. 6(a), a typical distribution testing feeder is under study considering DER penetration. Converter \#1 is connected at bus \#3 and converter \#2 is connected at bust \#5. Meanwhile, critical loads are connected at bus \#1 and \#4, respectively, while non-critical loads are connected at bus \#2,\#6,\#7 and \#8, respectively. As shown in Fig. 6(b), the dotted lines represent the communication network among all the buses. The looped-chain-based active current sharing scheme is applied in this test system to equalize the output current in converter $\# 1$ and $\# 2$. Meanwhile, the average-voltage-based secondary control is employed to restore the DC voltage of critical loads at bus \#1 and \#4. The system parameters are shown in Table 1. The layout of the MATLAB/Simulink model is shown in Fig. 7, and the control diagram is the same as in Fig. 4.

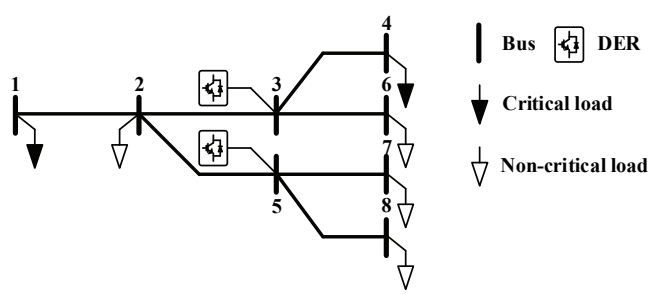

(a)

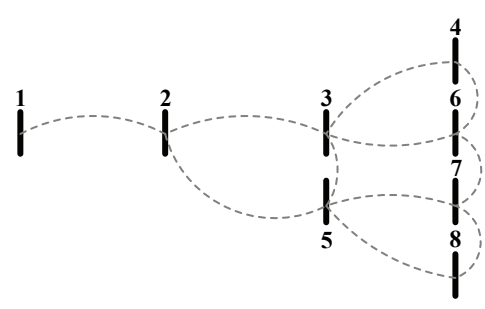

(b)

Fig. 6. Configuration of the test system: electrical connection (a); communication network (b)

Table 1. System parameters

\begin{tabular}{l|c}
\hline \multicolumn{2}{c}{ Control system } \\
\hline Average DC voltage controller & 0.5 \\
\hline$k_{\text {pavgv }}$ & 1 \\
\hline$k_{\text {iavgv }}$ & \\
\hline Average neighboring DC current controller & $1 \times 10^{-4}$ \\
\hline$k_{\text {pavgc }}$ & 6 \\
\hline$k_{\text {iavgc }}$ & \\
\hline Droop coefficient & 2 \\
\hline$R_{d}$ & \\
\hline Inner voltage controller & 0.2 \\
\hline$k_{\text {pinv }}$ & 1.6 \\
\hline$k_{\text {iinv }}$ & \\
\hline Inner current controller & 0.09 \\
\hline$k_{\text {pinc }}$ & 4 \\
\hline$k_{\text {iinc }}$ &
\end{tabular}




\begin{tabular}{lc}
\hline \multicolumn{2}{c}{ Main Power Circuit } \\
\hline Unit line resistance $(\Omega / \mathrm{km})$ & 0.3 \\
\hline Critical load resistance at node $1(\Omega)$ & 12 \\
\hline Critical load resistance at node $4(\Omega)$ & 16 \\
\hline Non-critical load resistance at node $2(\Omega)$ & 20 \\
\hline Non-critical load resistance at node $6(\Omega)$ & 18 \\
\hline Non-critical load resistance at node $7(\Omega)$ & 20 \\
\hline Non-critical load resistance at node $8(\Omega)$ & 16 \\
\hline Reference of DC output voltage $(\mathrm{V})$ & 100 \\
\hline DC input voltage $(\mathrm{V})$ & 60 \\
\hline $\begin{array}{l}\text { Filter capacitance of the interface } \\
\text { converter }(\mu \mathrm{F})\end{array}$ & 600 \\
\hline $\begin{array}{l}\text { Filter inductance of the interface } \\
\text { converter }(\mu \mathrm{H})\end{array}$ & 300 \\
\hline
\end{tabular}

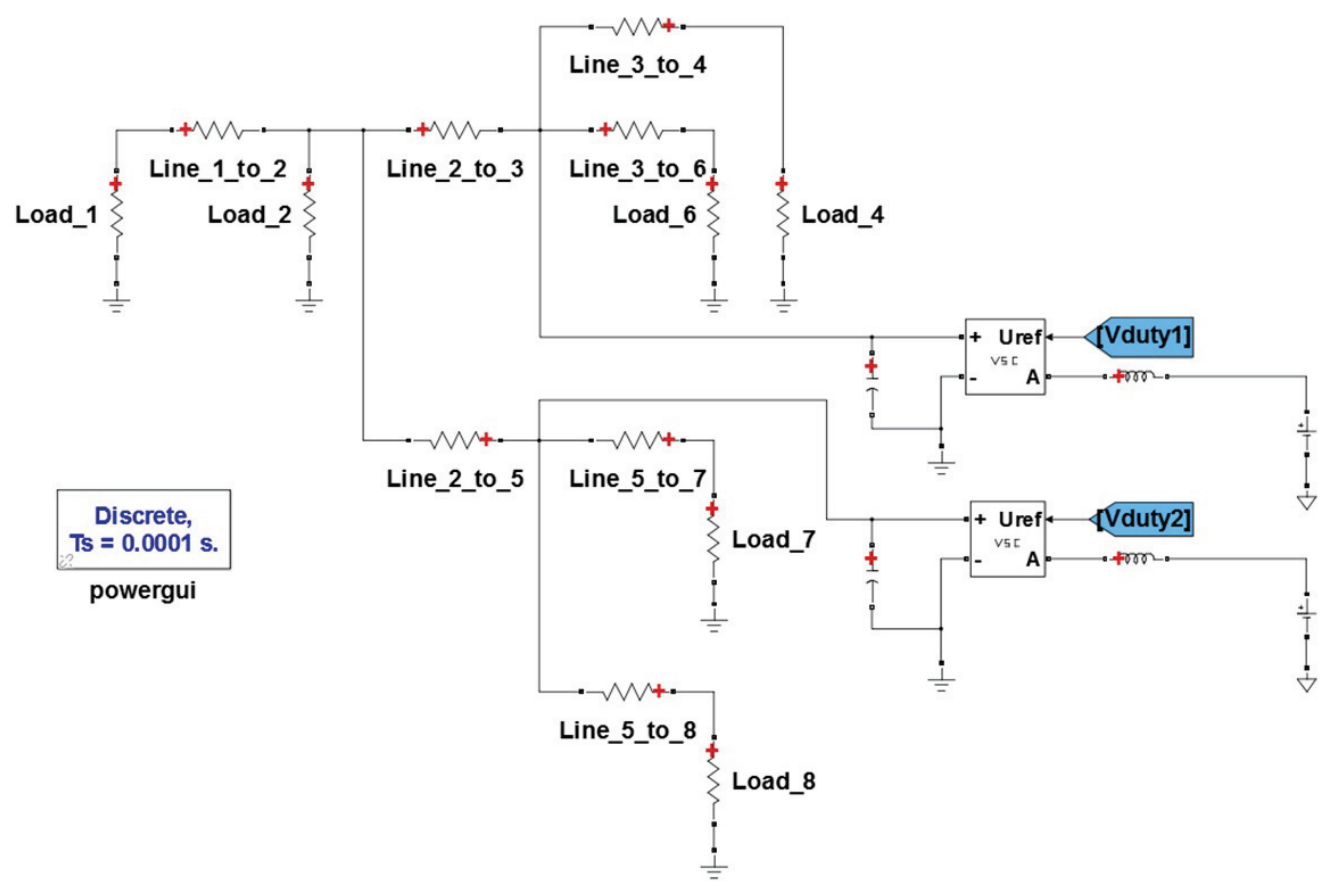

Fig. 7. Simulation layout in MATLAB/Simulink

Test case \#1: current sharing and voltage restoration in normal condition.

As shown in Fig. 8, in normal operation condition, no communication delays exist in the system. Hence, information exchange can be successfully achieved among all the neighboring buses. Here, the blue curve represents the output current or voltage of converter \#1, while the red curve represents the output current of converter \#2. By using the looped-chain-based 
active current sharing approach, the current difference can be minimized from $2.28 \mathrm{~A}$ to $0 \mathrm{~A}$, and the deviation of average voltage can be reduced from $3.9 \mathrm{~V}$ to almost zero. Since the critical loads are connected at bus \#1 and \#4, the proposed method is used to enhance the average DC bus voltage at these two buses and simultaneously increase current sharing accuracy.

Test case \#2: fault tolerant operation with communication loss.

As shown in Fig. 9(c), a communication loss occurs at $16 \mathrm{~s}$. This communication loss causes the communication channel between bus $\# 2$ and \#3 damaged. Hence, the voltage information at bus \#1 is transferred to bus \#3 following the path $1 \rightarrow 2 \rightarrow 5 \rightarrow 3$ rather than $1 \rightarrow 2 \rightarrow 3$.

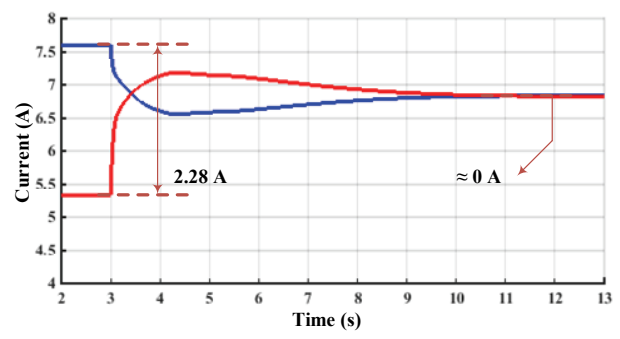

(a)

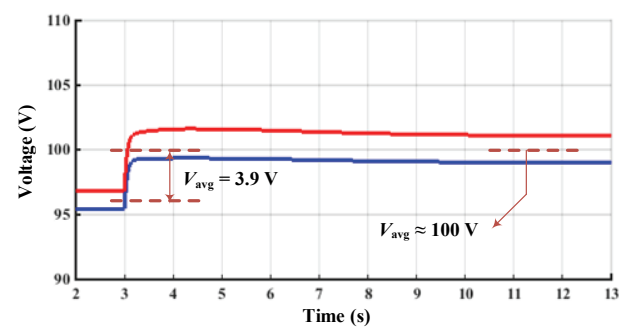

(b)

Fig. 8. Current sharing enhancement and voltage restoration in normal operation: current waveforms (a); voltage waveforms (b)

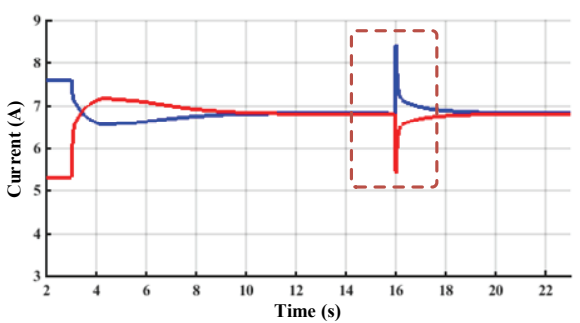

(a)

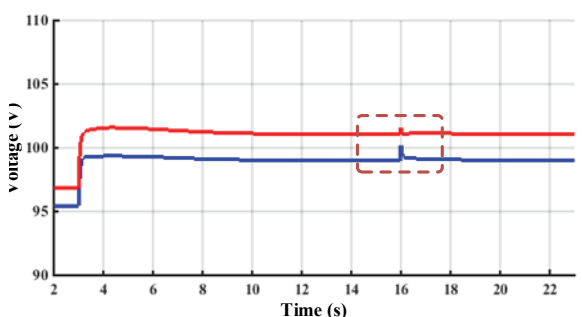

(b)

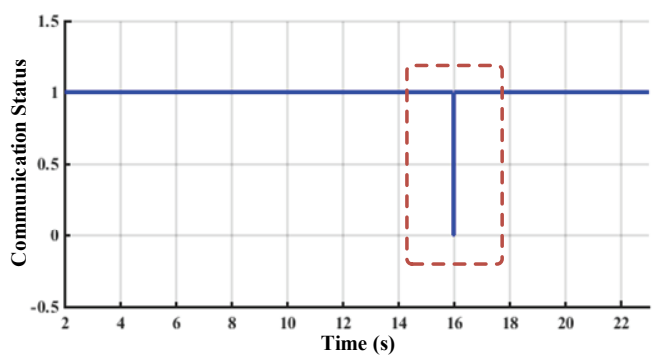

(c)

Fig. 9. Current sharing enhancement and voltage restoration with communication loss: current waveforms (a); voltage waveforms (b); communication status (c) 
Although there are some transient disturbances induced by this communication loss, the current sharing and the restoration of average DC voltage can still be achieved, as shown in Fig. 9(a) and (b).

Test case \#3: load change.

As shown in Fig. 10, when a step-change of the load current at bus \#1 occurs, the proposed looped-chain-based approach can still ensure equal current sharing by controlling the average current among neighboring buses. Meanwhile, the average DC bus voltage at the critical buses can also be restored.

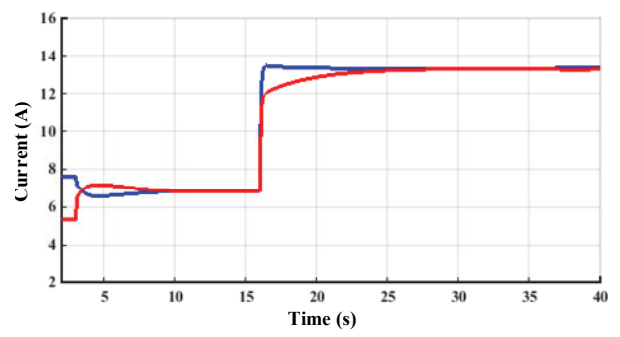

(a)

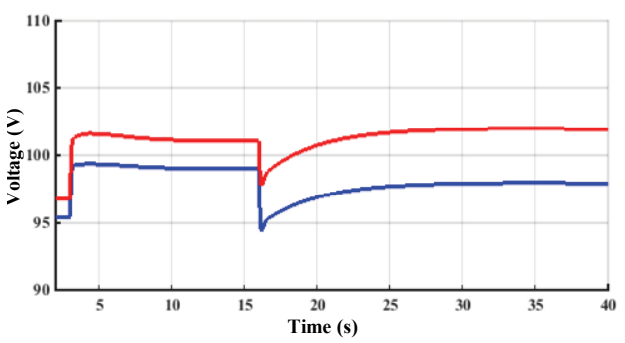

(b)

Fig. 10. Current sharing enhancement and voltage restoration with load change: current waveforms (a); voltage waveforms (b)

\section{Conclusion}

In this paper, a looped-chain-based active current sharing approach is proposed to ensure equal DC output current in each interface converter in DC microgrids. This current sharing scheme is realized based on the DC output current measurements from the neighboring interface converters. Since only one-side neighboring converters are needed for obtaining the DC output current, the dependency of the communication network is halved compared to conventional current sharing schemes. Meanwhile, since the proposed method is implemented in a distributed way, there is no central controller used in the system. Hence, the reliability of the whole system can be enhance. An improved secondary control scheme is implemented to restore the DC voltage at the buses with critical loads. Rather than restoring the DC voltage at one given bus, the average DC bus voltage is calculated using the complementary communication network so that the DC bus voltage at all the buses with critical loads can be restored. The selectivity of secondary control can be improved, and the average DC bus voltage can be successfully controlled to minimize the DC voltage deviation induced by droop control. The proposed control diagram has been tested in a DC microgrid with multiple buses to demonstrate the feasibility and applicability.

\section{Acknowledgement}

This paper is supported by the Fundamental Research Funds for the Central Universities, project number: 2572017BB13 


\section{References}

[1] Schenk T., Stokes L.C., The Power of Collaboration: Engaging All Parties in Renewable Energy Infrastructure Development, IEEE Power and Energy Magazine, vol. 11, no. 3, pp. 56-65 (2013).

[2] Arriaga M., Cañizares C.A., Kazerani M., Renewable Energy Alternatives for Remote Communities in Northern Ontario, Canada, IEEE Transactions on Sustainable Energy, vol. 4, no. 3, pp. 661-670 (2013).

[3] Damiano A., Gatto G., Marongiu I., Porru M., Serpi A., Real-Time Control Strategy of Energy Storage Systems for Renewable Energy Sources Exploitation, IEEE Transactions on Sustainable Energy, vol. 5, no. 2, pp. 567-576 (2014).

[4] Hatziargyriou N., Asano H., Iravani R., Marnay C., Microgrids, IEEE Power and Energy Magazine, vol. 5, no. 4, pp. 78-94 (2007).

[5] Morstyn T., Hredzak B., Demetriades G.D., Agelidis V.G., Unified Distributed Control for DC Microgrid Operating Modes, IEEE Transactions on Power Systems, vol. 31, no. 1, pp. 802-812 (2016).

[6] Augustine S., Mishra M.K., Lakshminarasamma N., Adaptive Droop Control Strategy for Load Sharing and Circulating Current Minimization in Low-Voltage Standalone DC Microgrid, IEEE Transactions on Sustainable Energy, vol. 6, no. 1, pp. 132-141 (2015).

[7] Liu Y., Wang J., Li N. et al., Enhanced Load Power Sharing Accuracy in Droop-Controlled DC Microgrids with Both Mesh and Radial Configurations, Energies, vol. 8, no. 5, pp.3591-3605 (2015).

[8] Lu X., Guerrero J.M., Sun K., Vasquez J.C., An Improved Droop Control Method for DC Microgrids Based on Low Bandwidth Communication With DC Bus Voltage Restoration and Enhanced Current Sharing Accuracy, IEEE Transactions on Power Electronics, vol. 29, no. 4, pp. 1800-1812 (2014).

[9] Moayedi S., Nasirian V., Lewis F.L., Davoudi A., Team-Oriented Load Sharing in Parallel $D C-D C$ Converters, IEEE Transactions on Industry Applications, vol. 51, no. 1, pp. 479-490 (2015).

[10] Nasirian V., Davoudi A., Lewis F.L., Guerrero J.M., Distributed Adaptive Droop Control for DC Distribution Systems, IEEE Transactions on Energy Conversion, vol. 29, no. 4, pp. 944-956 (2014).

[11] Roslan A.M., Ahmed K.H., Finney S.J., Williams B.W., Improved Instantaneous Average CurrentSharing Control Scheme for Parallel-Connected Inverter Considering Line Impedance Impact in Microgrid Networks, IEEE Transactions on Power Electronics, vol. 26, no. 3, pp. 702-716 (2011).

[12] Guerrero J.M., Vasquez J.C., Matas J., de Vicuna L.G., Castilla M., Hierarchical Control of Droop-Controlled AC and DC Microgrids-A General Approach Toward Standardization, IEEE Transactions on Industrial Electronics, vol. 58, no. 1, pp. 158-172 (2011). 